\title{
Access to Interpreter Services at U.S. Dental School Clinics
}

\begin{abstract}
Lisa Simon, DMD; Lauren Hum, BA; Romesh Nalliah, BDS
Abstract: The number of Americans with limited English proficiency (LEP) is growing, and legal protections mandate that LEP individuals have equal access to health care services. The aim of this study was to determine the availability of interpretation services in U.S. dental school clinics and the kinds of instruction dental students are given regarding treatment of LEP patients. A survey was distributed to the academic deans of all U.S. dental schools; 35 completed the survey for a response rate of $58 \%$. Respondents were asked to report on the number of LEP patients treated in their student clinics, the resources available to students working with LEP patients, and the extent of instruction offered. Descriptive statistics were calculated. The results indicated that the proportion of LEP patients treated at U.S. dental schools was perceived to be higher than that of the general population. The availability of interpreter services and the extent of student education about LEP individuals varied widely. Among the responding schools, the most common language spoken by LEP patients was Spanish, followed by Chinese (Mandarin and Cantonese) and Russian. Most of the responding dental schools reported offering fewer than two hours of instruction to their predoctoral students on treating LEP patients. Although almost $90 \%$ of the respondents indicated believing LEP patients received care equal in quality to that of non-LEP patients in their clinics, only $61.9 \%$ indicated that their students were adequately prepared to manage LEP patients following graduation. These findings suggest that dental schools should consider curricular innovations that will prepare students to work with LEP populations and improve the ability of LEP patients to receive care in the teaching clinic setting.
\end{abstract}

Dr. Simon is Instructor in Oral Health Policy and Epidemiology, Harvard School of Dental Medicine; Ms. Hum is a Class of 2016 DMD student, Harvard School of Dental Medicine; and Dr. Nalliah is Director of Clinical Education, University of Michigan School of Dentistry and an MS candidate, Harvard School of Public Health. Direct correspondence to Dr. Lisa Simon, Harvard School of Dental Medicine, 188 Longwood Avenue, Boston, MA 02115; 617-432-2917; Lisa_Simon@hsdm.harvard.edu.

Keywords: dental education, clinic management, clinical education, patient care management, health care disparities, cultural competence, limited English proficiency, interpreters

Submitted for publication 4/21/15; accepted 6/25/15

$\mathrm{R}$ ecently updated dental education standards of the Commission on Dental Accreditation (CODA) include competencies pertaining to the ability to sensitively and effectively treat individuals from diverse cultural groups and belief systems, including individuals with limited English proficiency (LEP). ${ }^{1}$ In 2011, 25.2 million LEP individuals resided in the United States, comprising 8.7\% of the population. ${ }^{2}$ LEP populations have increased dramatically in the past decades, and this growth is expected to continue. ${ }^{2}$ Modern dental practice will include the treatment of individuals with LEP, and dental school graduates must be competent to lead teams that provide holistic care to these individuals.

LEP patients have been found to be more likely to receive lower quality care and have poorer health outcomes than patients who speak English as a first language; however, their care is improved when they have access to professional interpreter services. ${ }^{3-5}$ Title VI of the 1964 Civil Rights Act mandates that no program receiving federal funding, including Medicaid payments, may restrict access to LEP individuals. ${ }^{6}$ Guidelines for The Joint Commission require hospital systems, which may include dental clinics, to provide interpreter services to all patients and families with limited English proficiency. ${ }^{7}$ However, enforcement of this legal obligation is inconsistent, many dental clinics do not offer access to formal interpreter services, and such services are even more uncommon in the private practice dental setting. ${ }^{89}$ The aim of this study was to evaluate and report the availability of interpreter services to students treating patients in U.S. dental school clinics and the extent of instruction students receive in this area.

\section{Materials and Methods}

The study protocol was reviewed and approved as "not human subjects research" by the Harvard Medical School/Harvard School of Dental Medicine Institutional Review Board (IRB14-0529). This was a cross-sectional survey-based study. The sample was comprised of the academic affairs deans or those in equivalent positions at U.S. dental schools. A dental school was considered eligible if it had been accredited by CODA.

Contact information was obtained from publicly accessible school websites for the 60 U.S. dental schools accredited by CODA at the time. Solicitation 
letters were emailed to the academic deans; a link to the survey was included in the invitation. A second letter was sent three weeks after the initial invitation

Table 1. Class size and region of participating dental schools, by number and percentage of total respondents $(n=35)$

\begin{tabular}{lc} 
Students/Region & Number (\%) \\
\hline Students per class & \\
80 or fewer & $13(37.1 \%)$ \\
$81-120$ & $18(51.4 \%)$ \\
$121-160$ & $3(8.6 \%)$ \\
$161-200$ & 0 \\
More than 200 & $1(2.9 \%)$ \\
Region & \\
Northeast & $5(14.3 \%)$ \\
Midwest & $12(34.3 \%)$ \\
South & $11(31.4 \%)$ \\
Southwest & $1(2.9 \%)$ \\
West & $6(17.1 \%)$ \\
\hline
\end{tabular}

Table 2. Limited English proficiency (LEP) patients in participating dental schools' clinics $(n=35)$

Question Number (\%)

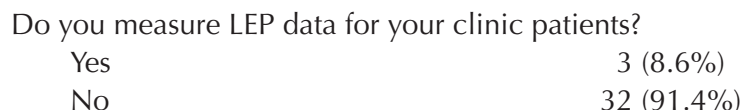

Estimated percentage of patients who have LEP

$\begin{array}{lc}\text { Range } & 1 \%-80 \% \\ \text { Average } & 22.97 \% \\ \text { Standard deviation } & 17.39\end{array}$

Most common primary languages of LEP patients ${ }^{\dagger}$ Spanish

$35(100.0 \%)$

Chinese

$12(34.3 \%)$

Mandarin

$4(11.4 \%)$

Cantonese

$1(2.9 \%)$

Russian

No dialect specified $\quad 7(20.0 \%)$

Arabic $\quad 5(14.3 \%)$

Korean $2(5.7 \%)$

Portuguese $2(5.7 \%)$

Somali $2(5.7 \%)$

Creole $1(2.9 \%)$

French $1(2.9 \%)$

Hmong $1(2.9 \%)$

Kurdish $1(2.9 \%)$

Polish $1(2.9 \%)$

Sudanese $\quad 1(2.9 \%)$

Ukrainian $1(2.9 \%)$

${ }^{\dagger}$ Respondents were asked to indicate the top three languages. to increase the response rate. Responses were received from October 20 to December 2, 2014. Recipients of the emails were asked to respond to the survey on behalf of their institution. The solicitation letter also requested that recipients forward the survey link to the clinical dean or other appropriate administrator if needed to answer questions regarding the patient population or curricula in their institutions.

The survey contained a total of 16 questions, but respondents received between 12 and 16 prompts depending on their responses and the internal logic of the survey. The survey asked respondents to describe their institutions' policies and protocols on interpreter use and management of LEP patients in student clinics, as well as information on how students were educated about interaction with LEP individuals.

Following data collection, quantitative statistics regarding class sizes and locations of respondents' institutions were recorded. For questions in which numerical responses between 0 and 100 were requested on a visual analog scale, range, mean, and standard deviation were calculated.

\section{Results}

Of the 60 academic deans invited to participate in the study, 41 agreed to participate and began the survey. A total of 35 respondents completed the survey $(58.3 \%)$. The size of each dental school class ranged from fewer than 80 students to greater than 200 (Table 1). However, smaller class sizes were more commonly represented. The majority of respondents indicated that their institutions had fewer than 120 students in each class. All regions of the continental United States were represented, although most respondents were at dental schools in the midwest (34.3\%) or southern United States (31.4\%).

The majority of respondents indicated their institutions do not record data on the prevalence of LEP patients, with only $8.6 \%$ indicating this information is routinely gathered (Table 2 ). The estimated prevalence of LEP patients at the clinics of responding dental schools ranged from $1 \%$ to $80 \%$, with a mean prevalence of $23.0 \%$ (SD 17.4\%). Respondents were prompted to list the three most frequently spoken languages of their institution's LEP patients. Among the respondents, 17 indicated fewer than three languages in their response. All respondents (100.0\%) listed Spanish as the most common primary language of LEP patients at their institution, with Chinese dialects (34.3\%), Russian (17.1\%), and Vietnamese (17.1\%) 
comprising the second, third, and fourth most commonly spoken languages, respectively.

Most respondents (82.9\%) indicated the presence of some formal curricular content for students in working with LEP patients at their institution (Table 3). If the respondents indicated that no formal instruction or resources were available, they were not prompted to answer additional questions about instruction and resources available for student training.

Of the responding institutions, $48.5 \%$ reportedly do not take language into account when assigning LEP patients to student clinicians (Table 4). If the institutions do not assign LEP patients to student providers in the same manner as English-speaking patients, the majority (88.2\%) said they attempt to pair the patient with a provider who speaks the same language or can speak a similar language. Five institutions (15.2\%) indicated that there are no formal interpreter services available on the teaching clinic floor. If no formal interpreter services for the LEP patient's primary language were available, respondents indicated that most often (71.4\%) an ad hoc interpreter affiliated with the school was utilized. No respondents indicated they refer LEP patients to an external clinic that offers interpreter services or care in the patient's primary language.

Among these respondents, $37.1 \%$ indicated that instruction for treating LEP individuals was directly integrated into their schools' curricula through either lecture or interactive content. Among institutions that offered instruction on managing LEP patients, $40.0 \%$ reported that only written resources were available for students, and $17.1 \%$ reported that no curricular content pertaining to LEP patients was available. The majority (59.3\%) of responding institutions with LEP curricula provided two or fewer hours of instruction on this topic. No responding institutions provided more than eight hours of formal instruction or training. A two-tailed t-test was conducted to compare mean reported prevalence of LEP patients between institutions with fewer than two hours of reported formal training and more than two hours (two to eight hours) of reported formal training in their predoctoral curricula, but no statistical significance was found $(\mathrm{p}=0.22)$.

The majority of the respondents said they believe that LEP patients receive sufficient quality of care and that students are prepared to provide care to LEP patients at their institutions. The respondents indicated a mean agreement of $88.8 \%$ on a $0-100$ visual analog scale (range 33\%-100\%, SD 16.3\%) with the statement "LEP patients receive the same quality of care as non-LEP patients in our teaching practices." The respondents indicated a mean agreement of $61.9 \%$ on the same scale with the statement "When students graduate from our institution, they are adequately prepared to independently manage LEP patients."

Table 3. Formal predoctoral instruction on treatment of limited English proficiency (LEP) patients, by number and percentage of responding schools $(n=35)$

Question

Number $(\%)$

Is there formal instruction and/or resources for dental students (e.g., lectures, videos, reading material, seminars, hands-on practice) in managing LEP patients?

Yes

Formal instruction and resources available

$29(82.9 \%)$

Formal instruction only

Resources available only

$13(37.1 \%)$

$2(5.7 \%)$

No

$14(40.0 \%)$

$6(17.1 \%)$

What is the primary method of instruction your institution uses to educate students on management of LEP patients?

Written content: provide materials and other resources to peruse

Lectures: provide educational information in class

$9(33.3 \%)$

Hands-on: provide opportunities to practice interaction and receive feedback

How many hours of formal instruction or training in managing LEP patients will students have had by the time they graduate from your institution?

Less than 2 hours

2-4 hours

$16(59.3 \%)$

4-6 hours

$5(18.5 \%)$

6-8 hours

$3(11.1 \%)$

$3(11.1 \%)$ 
Table 4. Protocols for management of limited English proficiency (LEP) patients in clinics, by number of respondents $(\mathbf{n}=35)$

Question

Number

Which statement best describes how LEP patients are assigned to student providers?

They are assigned through the same mechanisms as English-speaking patients.

16

They are paired with students who have indicated they speak the patient's primary language.

Other

If no student providers speak the primary language, how are LEP patients assigned?

We assign the patient randomly.

We assign the patient to a student provider who can speak a similar language.

Other

Which statement best describes the interpreter services available on the clinic floor?

We have formally trained interpreters employed by our institution whom student providers can request for appointments.

We subscribe to interpreter services provided via phone or Internet via programs like IPOP that are available in the clinic.

We have formal interpreter services available (in-person or via phone or Internet) for some but not all languages spoken by patients (please specify).

We do not have formal interpreter services available.

If the student provider and patient do not have access to formal interpreter services on the clinic floor for the patient's primary language:

Patients may bring an ad hoc interpreter they know personally (family member or friend).

Student providers and patients may utilize ad hoc interpreters affiliated with the school (staff, other students, faculty).

We refer the patient to an external site that has formal interpreter services.

Other

\section{Discussion}

The National Institutes of Health (NIH) define cultural competence as the ability of health care providers to "deliver services that are respectful of and responsive to the health beliefs, practices, and cultural and linguistic needs of diverse patients." 10 The most recent CODA standards include competencies related to cultural competence, noting that such training "stresses the importance of providers' understanding the relationship between diversity of culture, values, beliefs, behavior, and language and the needs of patients." ${ }^{\prime \prime}$ Although cultural competence curricula for predoctoral dental education have been described, ${ }^{11}$ competencies relating to working with LEP individuals are not explicitly mandated and may or may not be included. ${ }^{12}$

Working with LEP individuals is only one part of cultural competence; however, as LEP populations grow, it is increasingly important. LEP individuals comprised almost $9 \%$ of the U.S. population in 2011, an increase of $80 \%$ since $1990 .{ }^{2}$ Individuals with LEP are more likely to be uninsured and have a lower income. ${ }^{12}$ Oral health is also poorer on average in these populations. $^{3}$ The six states with the highest LEP populations (California, Texas, New York, Florida, Illinois, and New Jersey) also have 20 of the current 65 U.S. dental schools. ${ }^{2,13}$ The respondents to our survey estimated that the percentage of LEP patients seen in their dental school clinics was $22.4 \%$, suggesting this level may be nearly three times the national average. The demographics of patients seen at dental school clinics have not been well described in the literature, but a survey of California dental students found that $10-20 \%$ of their patients required an interpreter. ${ }^{14,15}$ Given these high rates of LEP patients, curricular competencies pertaining to these populations are critical for appropriate patient care.

The Civil Rights Act of 1964 mandates that all institutions receiving federal funding provide access to individuals regardless of language. ${ }^{6}$ This right to access was reinforced by Executive Order 13166, issued by President Clinton in 2000, with Policy Guidelines amended in 2003 under the Bush Administration. ${ }^{6}$ While these Policy Guidelines emphasized the legal obligation of federally funded institutions to provide equal access to LEP individuals, no specific legal guidelines exist; indeed, the most recent Policy Guidelines acknowledge that smaller institutions may 
have different accommodations for LEP individuals than larger institutions. ${ }^{6}$ Moreover, only 12 states and the District of Columbia provide reimbursement through Medicaid for interpreter services. ${ }^{15}$ It is perhaps unsurprising given these inconsistencies that one-third of North Carolina safety net dental providers were not aware of the legal mandate to provide access to LEP patients. ${ }^{8}$

Increased education and exposure to medical interpreters is correlated with increased facility with their use. ${ }^{5}$ However, the majority of respondents in our study indicated that students at their institutions receive less than two hours of instruction in working with LEP patients before graduation. This estimate does not include the time students may spend treating LEP patients during their clinical education. Research on education in interpreter use for medical students has found that even a two-hour intervention can significantly impact interpreter use by students. ${ }^{16}$ Curricula for medical students, medical residents, nursing students, and physician assistant students have been previously described. ${ }^{16-20}$ A notable point to consider is that, unlike the medical profession, dentists do not often practice within large organizations with broad support to help manage a variety of patients. In fact, $59 \%$ of dentists in the United States are in solo practice, which can be isolating and may not provide the clinician support mechanisms available in a hospital or larger group practice. ${ }^{21}$ Hence, predoctoral preparation of dental providers to manage LEP patients is critical.

Providers trained in adequate interpreter use are more likely to report self-efficacy in providing care to LEP patients and in knowing when interpreter use is indicated. ${ }^{22,23}$ To our knowledge, no curricula specifically for dental students have been published. Successful interventions have ranged from Internetbased modules to didactic sessions and objective structured clinical examinations (OSCEs). The current generation of dental students, the Millennials, have a documented preference for hands-on and interactive curricula rather than conventional didactic teaching methods. ${ }^{24,25}$ This preference may lead to opportunities for innovation, including incorporation of interpreter use and LEP competencies into emerging cultural competence curricula and the use of novel educational methodologies to transfer and retain this information in a new generation of learners. ${ }^{26-28}$

Beyond the clinical setting, international and community-based clinical experiences may increase student exposure to non-English-speaking and otherwise culturally diverse populations. Approximately half of all dental schools reported the presence of programs allowing dental students to provide care in an international setting in 2012, and many students express interest in working internationally while in training or after graduation. ${ }^{29,30}$ While such experiences may increase students' exposure to LEP or non-English-speaking patients, additional cultural competency curricula are necessary for students to make responsible contributions in the global setting. ${ }^{31}$

An additional means to increase dental student facility with LEP populations is through communitybased clinical experiences (CBCEs), in which students work in safety net settings that may have high numbers of LEP patients. ${ }^{32}$ CODA now recommends that all schools provide these experiences to their students, ${ }^{1}$ and in 201022 U.S. dental schools reported community rotations longer than 25 days. ${ }^{33}$ The populations served through these experiences vary widely among clinical sites, and not all underserved populations may be LEP. However, one study found that senior dental students who treated LEP patients while participating in CBCEs were more likely to report comfort treating LEP populations. ${ }^{34}$ It is important to note that while these experiences may impact students' ability to care for LEP patients in their future practice, changes in schools' clinics are necessary to accommodate LEP patients currently treated there.

Beyond formal curricula, the majority of respondents $(84.8 \%)$ in our study indicated that their students had access to formal interpreter services to communicate with their LEP patients while in clinic. This finding is in contrast to a 2009 survey of California dental schools, in which only ad hoc interpreters were reported to be in use. ${ }^{15}$ Use of interpreters improves LEP patients' satisfaction with their care and their health outcomes, although this practice has not been studied in the dental setting. ${ }^{5,35}$ Professionally trained medical interpreters are bilingual individuals who have additional training in health care terminology and cultural competence. Evolution of health care technology and telemedicine means that interpreter services may not require the physical presence of an interpreter. Videoconferencing services, telephone language lines, and interpreter phone-on-a-pole (IPOP) are alternative methods of providing interpreter services as needed. While Locatis et al. found that physicians reported a preference for in-person interpretation, there was no significant difference in patient satisfaction compared to telephone interpreter services. ${ }^{36}$ Moreover, both patients and caregivers rate professionally interpreted 
interactions, whether remote or in-person, as superior to interactions facilitated by an ad hoc interpreter. ${ }^{37}$ Remote interpreter services may also allow for use of an interpreter during interactions in which waiting for an interpreter to arrive is not practical.

In our study, 13 respondents indicated that ad hoc interpreters were used in their dental school clinics. Ad hoc interpreters also remain the most common form of interpretation among dentists; however, their use is associated with poorer outcomes, and they may lack necessary proficiency in translating technical terminology or in serving as a bridge between patient and clinician. ${ }^{9,37,38}$ The use of family members as ad hoc interpreters can be especially difficult when the medical or dental interview includes questions of a personal nature. Information transfer is also improved when professional interpreters are used, rather than bilingual friends or family members. ${ }^{39}$ It is troubling to report that five respondents in our study reported that interpreter services were not available at all to students in their clinics.

Institutions may pair LEP patients with students who speak the patients' preferred language, a practice indicated by $45.5 \%$ of respondents in our study. This practice poses an interesting dilemma for dental schools as it limits the exposure of students who speak only English to linguistically diverse patients. Referral to a student who does speak the language of the LEP patient would improve communication and, perhaps, ensure a more rapid and efficient delivery of care. However, the primary role of dental school is to provide a safe environment for students to develop the necessary skills to deliver care in the "real world." Those students without experience working with LEP populations may resist treating these groups in future practice. Our survey did not ask whether schools required students to prove proficiency in a language before being paired with LEP patients. Even if schools persist in assigning LEP patients to students who speak their language, students and providers may overestimate their proficiency and, as a result, fail to utilize additional resources to care for their LEP patients. ${ }^{38,40}$ Institutions should consider assessing students' proficiency if they are to care for LEP patients without an interpreter. In both situations, the availability of professional interpreter services would improve LEP patients' care and the educational experience of students.

A previous survey found that dental school leadership acknowledged the importance of training future dentists in cultural competence; in spite of this, only $37.8 \%$ of the responding academic deans at dental schools in the United States and Canada indicated that their institutions were prepared to implement the educational domain pertaining to the use of crosscultural communication models. ${ }^{11}$ In our survey, almost $90 \%$ of the respondents indicated that they believed LEP patients received care equal in quality to non-LEP patients in the dental school clinic, yet only $61.9 \%$ indicated that students were adequately prepared to manage LEP patients following graduation. Future dentists will most certainly work with LEP patients; dental schools should consider implementing structured instruction that ensures students graduate with the skills needed to effectively treat this growing population.

\section{Conclusion}

This study found that the responding academic deans perceived patients at their dental schools' clinics to have limited English proficiency at higher rates than the national average. The extent of education and availability of resources students have to treat these patients varied widely among these schools. Less than ideal interpretation practices occurred in almost all institutions surveyed. As dental education evolves, dental schools have the opportunity to improve health outcomes and oral health equity beyond the reaches of the dental school clinic. Adoption of dynamic and meaningful instruction emphasizing cultural competence and access to interpreter services is an important means of producing a future oral health workforce that is sensitive to the needs of the U.S. population and able to reduce disparities in oral health.

\section{REFERENCES}

1. Commission on Dental Accreditation. Accreditation standards for dental education programs. Chicago: American Dental Association, 2013.

2. Limited English proficiency individuals in the United States: number, share, growth, and linguistic diversity. Washington, DC: Migration Policy Institute, 2011.

3. Flores G, Tomany-Korman SC. The language spoken at home and disparities in medical and dental health, access to care, and use of services in U.S. children. Pediatrics 2008;121(6):1703-14.

4. Manson A. Language concordance as a determinant of patient compliance and emergency room use in patients with asthma. Med Care 1988;26:1119-28.

5. Flores G. The impact of medical interpreter services on the quality of health care: a systematic review. Med Care Res Rev 2005;62(3):255-99. 
6. Chen AH, Youdelman M, Brooks J. The legal framework for language access in health care settings: Title VI and beyond. J Gen Intern Med 2007;22(Suppl 2):362-7.

7. The Joint Commission. Advancing effective communication, cultural competence, and patient-andfamily-centered care. At: www.jointcommission.org/ Advancing Effective Communication/. Accessed 23 Feb. 2015.

8. Hammersmith KJ, Lee JY. A survey of North Carolina safety-net dental clinics' methods for communicating with patients of limited English proficiency. J Public Health Dent 2009;69(2):90-4.

9. Goldsmith C, Slack-Smith L, Davies G. Dentist-patient communication in the multilingual dental setting. Aust Dent J 2005;50(4):235-41.

10. National Institutes of Health. Clear communication: cultural competence. At: www.nih.gov/clearcommunication/ culturalcompetency.htm. Accessed 20 Feb. 2015.

11. Holyfield LJ, Miller BH. A tool for assessing cultural competence training in dental education. J Dent Educ 2013;77(8):990-7.

12. Association of Asian-Pacific Community Health Organizations. Health centers: improving health care access for limited English proficient patients. Oakland, CA: Association of Asian-Pacific Community Health Organizations, 2004.

13. American Student Dental Association. List of U.S. dental schools. At: www.asdanet.org/us-dental-schools.aspx. Accessed 22 Feb. 2015.

14. Makarem SC, Coe JM. Patient retention at dental school clinics: a marketing perspective. J Dent Educ 2014;78(11):1513-20.

15. Itaya LE, Glassman P, Gregorczyk S, Bailit HL. Dental school patients with limited English proficiency: the California experience. J Dent Educ 2009;73(9):1055-64.

16. Shriner CJ, Hickey DP. Teaching and assessing family medicine clerks' use of medical interpreters. Fam Med 2008;40(5):313-5.

17. Kalet A, Gany F, Senter L. Working with interpreters: an interactive web-based learning module. Acad Med 2002;77(9):927.

18. McEvoy M, Santos MT, Marzan M, et al. Teaching medical students how to use interpreters: a three-year experience. Med Educ Online 2009;14:12.

19. Fung CC, Lagha RR, Henderson P, Gomez AG. Working with interpreters: how student behavior affects quality of patient interaction when using interpreters. Med Educ Online 2010;15.

20. Marion GS, Hildebrandt CA, Davis SW, et al. Working effectively with interpreters: a model curriculum for physician assistant students. Med Teach 2008;30(6).

21. Guay AH, Wall TP, Petersen BC, Lazar VF. Evolving trends in size and structure of group dental practices in the United States. J Dent Educ 2012;76(8):1036-44.

22. Hernandez RG, Cowden JD, Moon M, et al. Predictors of resident satisfaction in caring for limited English proficient families: a multisite study. Acad Pediatr 2014;14(2):173-80.

23. Thompson DA, Hernandez RG, Cowden JD, et al. Caring for patients with limited English proficiency: are residents prepared to use medical interpreters? Acad Med 2013;88(10):1485-92.

24. Sanders J, Morrison C. What is the net generation? The challenge for future medical education. Med Teach 2007;29:85-8.

25. McAndrew M, Johnston AE. The role of social media in dental education. J Dent Educ 2012;76(11):1474-81.

26. Donate-Bartfield E, Lobb WK, Roucka TM. Teaching culturally sensitive care to dental students: a multidisciplinary approach. J Dent Educ 2014;78(3):454-64.

27. Evans L, Hanes PJ. Online cultural competency education for millennial dental students. J Dent Educ 2014;78(6):867-75.

28. Pilcher ES, Charles LT, Lancaster CJ. Development and assessment of a cultural competency curriculum. J Dent Educ 2008;72(9):1020-8.

29. Cohen LK, Valachovic RW. Students passionate for global health: dental schools beginning to respond. Compend Contin Educ Dent 2012;33(7):470-1.

30. Karim A, Mascarenhas AK, Dharamsi S. A global oral health course: isn't it time? J Dent Educ 2008;72(11): 1238-46.

31. Seymour B, Benzian H, Kalenderian E. Voluntourism and global health: preparing dental students for responsible engagement in international programs. J Dent Educ 2013;77(10):1252-7.

32. Formicola AJ, Bailit HL. Community-based dental education: history, current status, and future. J Dent Educ 2012;76(1):98-106.

33. Bailit HL. Organization and management of communitybased dental education programs: an overview from the dental pipeline program. J Dent Educ 2010;74(10 Suppl):S9-16.

34. Kuthy RA, Heller KE, Riniker KJ, et al. Students' opinions about treating vulnerable populations immediately after completing community-based clinical experiences. J Dent Educ 2007;71(5):646-54.

35. Karliner LS, Jacobs EA, Chen AH, Mutha S. Do professional interpreters improve clinical care for patients with limited English proficiency? A systematic review of the literature. Health Serv Res 2007;42(2):727-54.

36. Locatis C, Williamson D, Gould-Kabler C, et al. Comparing in-person, video, and telephonic medical interpretation. J Gen Intern Med 2010;25(4):345-50.

37. Nápoles AM, Santoyo-Olsson J, Karliner LS, et al. Clinician ratings of interpreter-mediated visits in underserved primary care settings with ad hoc, in-person professional, and video conferencing modes. J Health Care Poor Underserved 2010;21(1):301-17.

38. Moreno MR, Otero-Sabogal R, Newman J. Assessing dual-role staff-interpreter linguistic competence in an integrated health care system. J Gen Intern Med 2007;22(Suppl 2):331-5.

39. Rosenberg E, Seller R, Leanza Y. Through interpreters' eyes: comparing roles of professional and family interpreters. Patient Educ Couns 2008;70(1):87-93.

40. Lion KC, Thompson DA, Cowden JD, et al. Impact of language proficiency testing on provider use of Spanish for clinical care. Pediatrics 2012;130(1):80-7. 